\title{
RESUMOS DE DISSERTAÇÕES E TESES
}

\author{
Resumos impressos de acordo com os originais enviados \\ pelas respectivas Coordenadorias de Pós-Graduação.
}

TíTULO: Diagnóstico Geoambiental de Zonas de Manguezal do Estuário do Rio Itanhém, Município de Alcobaça Região Extremo Sul do Estado da Bahia

AUTOR(A): Bárbara Rosemar Nascimento de Araújo

DATA: $\quad 31 /$ janeiro/2000

LOCAL: $\quad$ Universidade Federal da Bahia, Instituto de Geociências, Departamento de Geoquímica e Meio Ambiente, Salvador-BA

NÍVEL: $\quad$ Mestrado

BANCA EXAMINADORA: Antônio Fernando de Souza Queiroz/ IGEO/UFBA (Orientador) João Lamarck Argôlo /IGEO/UFBA

Ilson Guimarães Carvalho /IGEO/UFBA

Juarez Jorge Santos /IBIO/UFBA

Raimundo Donato Damasceno - UFF

RESUMO - Coletas de sedimentos, de folhas de Avicennia schaueriana Stapf e Leechn e de folhas de Avicennia germinans (L.) Stearn, foram realizadas em 7 estações, no manguezal do estuário do rio Itanhém, Alcobaça-BA. As estações 1 e 2 foram próximas à desembocadura do rio e da sede do município de Alcobaça. As estações 3 e 4, em sua porção intermediária. As estações 5, 6 e 7 na parte superior do rio, em direção à montante. Em cada estação foram feitas as seguintes coletas: testemunhos de sedimento de aproximadamente $1 \mathrm{~m}$ de profundidade, os quais foram seccionados em 5 partes de $20 \mathrm{~cm}$; folhas adultas a partir do $3^{\circ}$ nó, de Avicennia e foram determinados parâmetros físico-químicos "in situ": pH; temperatura e extinção da luz, nas águas superficiais adjacentes a cada estação. No sedimento foram determinados: as frações granulométricas, através do método da EMBRAPA; o teor de matéria orgânica, através do método gravimétrico e o teor de nitrogênio, pelo método Kjeldahl. No sedimento e nas folhas foram realizadas determinações dos teores de metais ( $\mathrm{Al}, \mathrm{Fe}, \mathrm{Mn}, \mathrm{Cu}, \mathrm{Pb}, \mathrm{Zn}, \mathrm{Cd}$ e $\mathrm{Cr}$ ), e de nutrientes ( $\mathrm{Na}, \mathrm{K}, \mathrm{Ca}$ e $\mathrm{Mg}$ ), através da espectrofotometria de absorção atômica, enquanto que o $\mathrm{P}$ foi determinado através de espectrofotometria visível. Exemplares de folhas foram ainda cortados transversalmente e paradermicamente e os tecidos foram evidenciados por coloração com verde iodo, vermelho congo e safranina. Além disso, foram realizados testes histoquímicos, para identificação de substâncias ergásticas. As análises evidenciaram que o sedimento é composto por 4 frações granulométricas argila, silte , areia fina e areia grossa, e que essas frações apresentaram uma assimetria positiva a muito positiva, indicando que o sedimento é formado por granulação fina, característicos de áreas de baixa energia. $\mathrm{O}$ teor de matéria orgânica e a relação $\mathrm{C} / \mathrm{N}$ foi elevada, principalmente nas estações $1 \mathrm{e} 2$, provavelmente devido a forte influência dos efluentes orgânicos urbanos e industriais despejados pelo município. Os elevados teores dos metais $\mathrm{Fe}$ e $\mathrm{Al}$ do substrato, provavelmente, é consequiência da composição mineralógica, composta principalmente por biotita, hornblenda, feldspato e muscovita. As altas correlações positivas do $\mathrm{Al}$ com o $\mathrm{Cu}, \mathrm{Zn}$ e o $\mathrm{Cr}$, indicam que a principal origem desses está no intemperismo dos minerais. O Zn permanece no substrato, devido a processos geoquímicos de co-preciptação, 
com oxi-hidróxidos de Fe e Mn. As altas correlações do Cr com o carbono orgânico, nitrogênio e fração argilosa, indicam que o Cr está sendo acumulado no sedimento seja por processos de complexação pela matéria orgânica e/ou por processos de adsorção/absorção pelas argilas. O teor de $\mathrm{Cd}$ medido no substrato esteve abaixo do limite de detecção da metodologia utilizada para análise. $\mathrm{O} \mathrm{Pb}$ apresentou teores considerados elevados para o meio ambiente, devido provavelmente a influência da combustão de veículos automotores como barcos de pesca e de turismo, carros e caminhões utilizados nas proximidades. As correlações obtidas entre o $\mathrm{Pb}$ e o Fe e $\mathrm{Mn}$, indicaram que o $\mathrm{Pb}$ pode estar sendo acumulado no substrato através de processos de co-preciptação, juntamente com oxi-hidróxidos de Fe e Mn. Teores elevados de $\mathrm{Na}, \mathrm{K}, \mathrm{Ca}$ e $\mathrm{Mg}$ e as correlações positivas entre sí no substrato provavelmente estão relacionados ao aporte de água do mar. A forte relação do $\mathrm{Mg}$ e do $\mathrm{P}_{2} \mathrm{O}_{5}$ com elementos representantes do material orgânico, indica uma significativa associação da matéria orgânica com o $\mathrm{Mg}$ e o $\mathrm{P}_{2} \mathrm{O}_{5}$ A folhas de Avicennia não apresentaram teores considerados elevados de $\mathrm{Fe}, \mathrm{Al}, \mathrm{Mn}, \mathrm{Cu}, \mathrm{Zn}, \mathrm{Cd}$ e $\mathrm{Cr}$. Entretanto, os teores de $\mathrm{Pb}$ foram bastante expressivos, podendo estar relacionado a absorção, associada a fixação desse elemento nas placas de ferro das raízes. Os nutrientes $\mathrm{K}, \mathrm{Ca}, \mathrm{Mg}, \mathrm{Na}$ e $\mathrm{P}_{2} \mathrm{O}_{5}$ nas folhas, apresentaram-se dentro das concentrações consideradas normais através de comparações com padrões. O alto teor de Na nas estações 1 e 2, devem estar relacionados a influência direta das águas do mar. A análise da anatomia das folhas do gênero Avicennia não evidenciou modificações estruturais nos tecidos. A presença de estruturas análogas a "lenticelas", nas folhas de $A$. germinans, não é relatada em referências anteriores.

Palavras-chave - Geoquímica, Manguezal, Anatomia vegetal, Substrato, Folhas de Avicennia

\section{Agência(s) Financiadora(s): CAPES, CADCT-BA}

TÍTULO DA DISSERTAÇÃo: Fitogeografia dos gêneros Jacaranda Juss. e Tabebuia Gomes ex DC. no bioma Cerrado

\section{AUTOR: \\ DATA DA DEFESA: \\ LOCAL DA DEFESA:}

\section{NÍVEL:}

BANCA EXAMINADORA:

\section{Rosana Farias}

30/agosto/2000.

auditório do Instituto de Biologia da UnB, Universidade de Brasília, Brasília, DF.

Mestrado

Prof $^{a}$. Dr ${ }^{a}$ Carolyn Elinore Barnes Proença (orientadora), Prof ${ }^{a} \operatorname{Dr}^{\mathrm{a}}$ Jeanine Maria Felfili Fagg e Prof ${ }^{\circ}$ Dr. Paulo José Fernandes Guimarães.

RESUMO - O trabalho aborda a distribuição fitogeográfica dos gêneros Jacaranda e Tabebuia (Bignoniaceae) no Cerrado contínuo no Brasil Central. A importância deste é o conhecimento da distribuição das espécies dos referidos gêneros, principalmente o local de ocorrência das espécies endêmicas, visando subsidiar, junto a outros táxons, novas áreas de preservação no bioma Cerrado. Para a correta distribuição das espécies realizaram-se dois trabalhos: 1) Florística, com confirmação, identificação, produção de chave e descrição diagnóstica das espécies, e 2) Fitogeográfica, com mapeamento das espécies no bioma Cerrado. Para a análise do Cerrado usou-se uma malha de $1^{\circ}$ x $1^{\circ} 30^{\prime}$, os retângulos foram agrupados com UPGMA utilizando-se o Índice de Similaridade de Jaccard. Os resultados da florística apresentaram 21 espécies de Jacaranda (2 destas são novas) e 14 espécies de Tabebuia. Os mapas de distribuição das espécies mostraram que estas são amplamente distribuídas na América do Sul (39\%) e em outras áreas do Brasil além do Cerrado (29\%), com apenas 6\% destas alcançando a América Central e o México. Uma significativa porcentagem (26\%) das espécies são restritas ao bioma Cerrado. A análise de UPGMA para os 27 retângulos bem coletados mostrou que existem 6 áreas fitogeográficas de Jacaranda/Tabebuia no Cerrado. Porém, existem áreas com esforço de coleta insuficiente para entrar na análise onde ocorrem as espécies J. praetermissa e Jacaranda sp1, podendo existir áreas adicionais.

Palavras-chave - florística, taxonomia, Brasil, Bignoniaceae, Tecomeae, savana.

Agência(s) Financiadora(s): CAPES 


\title{
TÍTULO DA DISSERTAÇÃO: Loranthaceae e Viscaceae no Bioma Cerrado
}

\author{
AUTOR: \\ Maria Aglaene Barboza \\ DATA DA DEFESA: \\ 31 de agosto de 2000. \\ NÍVEL: \\ Mestrado

\section{BANCA EXAMINADORA:} \\ Nome da Orientadora: \\ Nome dos Membros da Banca: Manoel Cláudio da Silva Júnior - UnB \\ Paulo José Fernandes Guimarães - UnB
}

RESUMO - Este trabalho tem como objetivo buscar subsídios para definir padrões fitogeográficos do Bioma Cerrado. O estudo apresentado baseou-se nas coleções botânicas dos principais herbários do Bioma Cerrado. Consta de dois capítulos: 1) Sinopse de Loranthaceae e Viscaceae representando o primeiro estudo destas famílias para o Bioma Cerrado. Foram encontrados 73 espécies e oito gêneros: Dendrophthora Eichl.; Phoradendron Nutt.; Oryctina Eichl.; Oryctanthus (Gris.) Eichl; Phthirusa Linn.; Psittacanthus Mart.; Struthanthus Mart. e Tripodanthus Van Tiegh.; 2) Fitogeografia das Loranthaceae/Viscaceae no Bioma Cerrado. As coordenadas geográficas das coletas foram registradas em planilha Excel e confeccionados mapas pelo Sistema de Informações Geográficas Maptitude. Foi construída uma matriz de similaridade utilizando o coeficiente de Jaccard. Os retângulos foram agrupados pela técnica de UPGMA (Unweighted Pair Groups Method using Arithmetic Averages), e produziu seis províncias para o Bioma Cerrado. Loranthaceae/Viscaceae apresentou padrões de distribuição geográfica que variam desde espécies amplamente distribuídas dentro e fora do Bioma Cerrado, até endêmicas restritas.

Palavras-chave-Flora, taxonomia, fitogeografia, padrões de distribuição.

\begin{abstract}
The aim of this study to seek subsidies to define phytogeografic patterns in the Cerrado Biome of Brazil. The study was based on the botanic collections of the main herbaria from the Cerrado Biome. It consists of 2 chapters: 1) Loranthaceae and Viscaceae synopsis representing the first studies of these families for the Cerrado Biome. Seventy-three species were found and 8 genera: Dendrophthora Eichl.; Phoradendron Nutt.; Oryctina Eichl.; Oryctanthus (Gris.) Eichl. ; Phthirusa Linn.; Psittacanthus Mart.; Struthanthus Mart.; Tripodanthus Van Tiegh.; 2) Phytogeography of the Loranthaceae / Viscaceae in the Cerrado Biome. The geographic coordinates were obtained from the 17 Brazilian herbarium and registered in an Excel table; maps were then produced by the Maptitude Geographic Information System. A similarity matrix was built using the Jaccard coefficient. The rectangles were clustered using UPGMA (Unweighted Pair Groups Method using Arithmetic Averages). This technique produced six provinces for the Cerrado Biome. Loranthaceae and Viscaceae showed patterns of geographic distribuition that vary from widely distributed species, in and out of the Cerrado Biome, to restricted endemics.
\end{abstract}

Key words - Flora, Phytogeography, Distribution patterns, Taxonomy

TÍTULO DO TRABALHO: Biologia Floral de Cuphea melvilla Lindlay (Lythraceae) na Estação Ecológica do Panga em Uberlândia-MG.

AUTORA: $\quad$ Ana Flávia de Oliveira Melazzo

DATA DE DEFESA: $\quad 17 / \mathrm{março/2000}$

LOCAL: $\quad$ Universidade de Brasília, Instituto de Ciências Biológicas, Pós-graduação Botâni ca, Brasília, DF. 


\author{
NÍVEL: $\quad$ mestrado \\ BANCA EXAMINADORA: Orientador: Prof. Dr. Paulo Eugênio Alves Macedo de Oliveira, UFU \\ Membro: Dra. Taciana Barbosa Cavalcanti, Embrapa-Cenargen \\ Membro: Prof ${ }^{a}$. Dra. Mariluza Granja e Barros, UnB
}

RESUMO - Estudou-se duas populações de Cuphea melvilla Lind. (Lythraceae) em solos hidromórficos nas bordas das matas de galeria do córrego do Panga, na Estação Ecológica do Panga em Uberlândia-MG para investigar a biologia floral e reprodutiva desta espécie, avaliando-se sua importância para a área de ocorrência e identificando-se os beija-flores visitantes.

Observações realizadas de janeiro a junho de 1999, fazendo-se visitas periódicas pela manhã e, algumas vezes, até o início da tarde, com idas a campo que variaram de uma a quatro visitas por semana ao longo de cinco meses.

Cuphea melvilla é uma espécie arbustiva apresentando características morfológicas e eventos florais que confirmam adaptação à polinização por beija-flores.

Floração longa iniciando-se na estação chuvosa e terminando no início da seca. Esta espécie mostrou-se autocompatível, formando frutos por polinizações cruzadas e autopolinizações manuais; e não apomítica necessitando de polinização para formar frutos.

Phaethornis pretrei, Amazilia fimbriata, Thalurania furcata, Eupetomena macroura e Chlorostolbon (fêmea) foram os beija-flores observados na planta, e todos realizaram visitas legítimas. Amazilia fimbriata e C. aureoventris apresentaram comportamento territorial em épocas diferentes, enquanto que $P$. pretre $i$ apresentou forrageamento por linhas de captura e $T$. furcata e $E$. macroura apresentaram forrageamento do tipo oportunístico.

Amazilia fimbriata, $C$. aureoventris e $P$. pretrei foram considerados os polinizadores potenciais.

Palavras-chave - Biologia floral, Lythraceae, beija-flores

ABSTRACT - Two populations of Cuphea melvilla Lind. (Lythraceae) occurring on hydromorphic soils on the edge of Panga's river gallery forest, in The Panga Ecological Station, Uberlândia-MG, were studied for many aspects of their floral and reproductive biology and their 2 importance for the species of hummingbird identified in the area.

Observations were carried out during over the flowering season of 1999 with periodic visits during the morning period and sometimes until the beginning of the afternoon. Field work effort varied between one to four visits per week during five months.

Cuphea melvilla is a shrub species that shows morphological adaptation to hummingbird's pollination and a long flowering season that begins on the rain season and finish on the beginning of the dry season. This species is selfcompatible forming fruits by cross-pollination and self-pollination but it is non-apomictic and need pollination to form fruits.

Pheathornis pretrei, Amazilia fimbriata, Thalurania furcata, Eupetomena macroura and Chlorostilbon aureoventris (female) were the hummingbirds observed visiting the $C$. melvilla's flowers and all realized legitimate visits to the flowers. A. fimbriata and $C$. aureoventris (female) showed territorial behaviour but in different seasons whereas $P$. pretrei appears to be trapliner. Finally, T. furcata and E. macroura showed opportunistic behaviour. Amazilia fimbriata, C. aureoventris and $P$. pretre $i$ were considered potentials pollinators.

Key words - Floral biology, Lythraceae, hummingbirds

Agência(s) Financiadora(s): CAPES

TÍTULO DO TRABALHO: Biologia Reprodutiva e Polinização em Melastomataceae no Parque do Sabiá, Uberlândia, MG.

AUTORA:

CURSO:

DATA DA DEFESA:

LOCAL:
Simone Carolina Sousa e Silva

Ciências Biológicas

04/agosto/2000

Universidade de Brasília, Instituto de Ciências Biológicas, Departamento de Botânica, Brasília, DF 
NÍVEL: $\quad$ Mestrado

BANCA EXAMINADORA: Prof. Dr. Paulo Eugênio A. M. Oliveira - UnB (Orientador) Prof ${ }^{a}$. Dr. ${ }^{a}$ Mariluza Granja Barros - UnB (Membro)

Prof. Dr. Paulo José Fernandes Guimarães - UnB (Membro)

RESUMO - Estudos sobre a biologia reprodutiva e polinização de Miconia chamissois Naud e Tococa formicaria Mart., Trembleya parviflora (D. Don) Cogn. e Rhynchantera grandiflora (Aubl.) DC., foram desenvolvidos no Parque do Sabiá, em Uberlândia, MG e foram baseados na fenologia de floração, caracterização morfológica, sistema de polinização, análise de viabilidade polínica, nos resultados de polinizações controladas e análise do crescimento do tubo polínico. Para Miconia cuspidata Naud., Miconia theazans (Bonpl.) Cogn., Miconia pseudonervosa Cogn., Lavoisiera imbricata (Thunb.) DC. e Desmocelis villosa (Aubl.) Naud. foram realizados apenas estudos sobre fenologia e viabilidade polínica. A fenologia de floração demonstra que as espécies florescem ao longo de todo o ano. O sistema de polinização mostrou que a antese é diurna. Todas as espécies oferecem como recompensa aos polinizadores pólen e a polinização é realizada por abelhas capazes de vibrar as anteras para a retirada do pólen ("buzz pollination"). Em relação à viabilidade polínica, apenas Miconia cuspidata apresentou pólen estéril. As demais apresentaram valores altos de viabilidade. O sistema reprodutivo mostrou-se diversificado. As espécies Miconia chamissois e Tococa formicaria mostraram auto-incompatibilidade ao nível do estilete. Rhynchanthera grandiflora é uma espécie autocompatível enquanto que Trembleya parviflora é apomítica facultativa.

Palavras-chave-Melastomataceae, biologia reprodutiva, agamospermia, polinização vibrátil.

Agência(s) Financiadora(s): CAPES

TíTULO DO TRABALHO: Gramíneas (Poaceae) da Área de Relevante Interesse Ecológico (ARIE) Santuário de Vida Silvestre do Riacho Fundo, DF - Brasil.

AUTOR:

Robson Rodrigues-da-Silva.

DATA DA DEFESA:

29/Novembro/2000.

LOCAL:

Universidade de Brasília, Departamento de Botânica. Brasília - DF.

NÍVEL:

Mestrado.

BANCA EXAMINADORA: Dr. Tarciso S. Filgueiras - Reserva Ecológica do IBGE.

Dr. José Ângelo Rizzo - UFG.

Dra ${ }^{\mathrm{a}}$. Carolyn Elinore Barnes Proença - UnB.

RESUMO - Neste trabalho relata-se o levantamento florístico das espécies de Poaceae da Área de Relevante Interesse Ecológico (ARIE) Santuário de Vida Silvestre do Riacho Fundo, DF - Brasil. Foram encontradas 107 espécies, distribuídas em 40 gêneros, sendo 82 nativas e 25 exóticas. São apresentadas chaves de identificação para gêneros e espécies, bem como descrições diagnósticas dos mesmos. As descrições das espécies são acompanhadas por comentários, incluindo habitat, potencial forrageiro, caracteres morfológicos marcantes ou outros aspectos de interesse botânico. Campo Úmido e Áreas Antrópicas foram os ambientes que apresentaram o maior número de espécies (33 e 31 espécies respectivamente). Coelorachis aurita (Steud.) A. Camus é citada pela primeira vez para a flora do Distrito Federal. Arthropogon filifolius Filg., encontrada em área de Campo Úmido, consta da lista de espécies raras no Distrito Federal. Comenta-se sobre as áreas degradadas do Santuário e apresenta-se sugestão para a reabilitação ecológica das mesmas. Comenta-se também sobre a população de capivara (Hydrocaeris hydrocaeris) e as possíveis consequiências do sobrepastejo destes grandes roedores sobre a flora graminóide.

Palavras-chave - florística, pastagens nativas, áreas degradadas, capivara. 
TITULO DA DISSERTAÇÃO:

NOME:

DATA DA DEFESA:

LOCAL:

NÍVEL:

ORIENTADOR:

BANCA EXAMINADORA:
“Aspectos da Reprodução em Brachiaria brizantha cv. Marandu".

Elizangela Ribeiro Alves.

23 de agosto de 2000.

Universidade de Brasília, Departamento de Botânica, Brasília, DF.

Mestrado.

Dra. Ana Cláudia Guerra de Araújo Embrapa-Recursos Genéticos e Biotecnologia.

Dra. Vera Tavares de Campos Carneiro Embrapa-Recursos Genéticos e Biotecnologia.

Prof. Dra. Mariluza Araújo Granja e Barros UnB.

RESUMO - A reprodução de plantas se dá pelo modo sexual ou assexual. A apomixia é um modo de reprodução assexual por sementes. Plantas que se reproduzem por apomixia geram embriões idênticos à planta-mãe, já que o embrião se desenvolve através da partenogênese de uma célula não reduzida. Esta característica é considerada extremamente importante para a agricultura, pois uma vez introduzida em genótipos de elite proporcionará a sua perpetuação. Nas plantas apomíticas pseudogâmicas, o desenvolvimento do endosperma é dependente da fertilização do núcleo polar enquanto nas apomíticas autônomas o endosperma desenvolve-se autonomamente. Neste trabalho, aspectos biológicos envolvidos no sucesso reprodutivo de um dos acessos apomíticos de Brachiaria brizantha, o cv. Marandu, foram avaliados através de estudos de biologia floral, observações morfológicas e análise citogenética. B. brizantha cv. Marandu apresenta estratégias reprodutivas similares a gramíneas em geral, com independência de polinizadores biológicos e indícios de xenogamia facultativa. O processo de formação e maturação do pólen é morfologicamente regular, resultando numa boa viabilidade polínica que descarta a ocorrência de macho-esterilidade, descrita para várias plantas apomíticas. O desenvolvimento inicial do embrião e degeneração de sinérgides independem da fertilização, sugerindo que a embrionia precoce pode facilitar a ocorrência da partenogênese. Morfologicamente, o desenvolvimento partenogenético do embrião é similar ao do zigótico em outras gramíneas. Pseudogamia foi caracterizada, o gameta masculino que fertiliza o núcleo polar para a formação do endosperma é reduzido e proveniente do mesmo acesso. A razão da contribuição genômica materna e paterna é $4 \mathrm{~m}: 2 \mathrm{p}$, similar a 2:1 encontrada nas plantas sexuais. A ocorrência de poliembrionia é baixa e parece não afetar a taxa de germinação dos embriões. Contudo, a taxa de formação de plantas originadas dessas sementes é menor do que nas monoembriônicas. $\mathrm{O}$ entendimento dos processos morfológicos relacionados ao modo de reprodução apomítico é de grande importância para o direcionamento de outros estudos que venham colaborar para o entendimento da apomixia e também dos processos reprodutivos em geral.

Palavras-chave-Poaceae, apomixia, fertilização, endosperma.

ABSTRACT - Two modes of reproduction are found in plants, sexual and asexual. Apomixis is an asexual reproduction mode through seeds. Apomictic plants give rise to embryos identical to the mother plant through parthenogenesis of a non-reduced egg cell. This feature is very important to agriculture, once incorporated in genotypes of agronomic importance, it allows its perpetuation. In pseudogamic apomictic plants, endosperm development depends on polar nuclei fertilization, while in autonomous apomicts, the endosperm develops autonomously. In this work, biological aspects of the reproductive success of apomictic accession Brachiaria brizantha cv. Marandu was analyzed. Studies of flowering biology aspects, morphological and cytogenetic analysis were performed. Similar reproductive strategies were observed in this accession, such as independence of biological pollinators and possible facultative xenogamy. Pollen grain development is morphologically regular, resulting viable pollen grains. These results found in $B$. brizantha $\mathrm{cv}$. Marandu deny the presence of male sterility, reported for several apomictic plants. Early embryo development and synergids degeneration are fertilization independent events, suggesting that precocious embryony may contribute to the occurrence of parthenogenesis. Parthenogenetic embryo development is morphologically similar to the zygotic embryo of other grasses. Pseudogamy was characterized, the polar nucleus is fertilized by reduced male sperm from the same accession. Therefore, the rate of maternal and paternal genomic contribution is $4 \mathrm{~m}: 2 \mathrm{p}$, similar to $2: 1$ found in sexual plants. Poliembyony occurs at low rates and does not affect germination level. The rate of plant development in 
polyembryonic seeds is lower than those in monoembryonic seeds. The comprehension of the morphological processes associated with apomictic reproduction is very important to direct other studies in the understanding of apomixis as well as reproductive process in general.

Key words - Poaceae, apomixis, fertilization, endosperm.

Agência(s) Financiadora(s): CAPES.

TÍTULO:

AUTORA:

DATA DA DEFESA:

LOCAL:

NÍVEL:

BANCA EXAMINADORA: Nome do orientador: Linda Styer Caldas, UnB

Nome do membro da banca: João Aguiar Nogueira Batista, Embrapa Recursos

Genéticos;

Nome do membro da banca: Mirian T. S. da Eira, Embrapa Recursos Genéticos; Nome do membro da banca: Rui Américo Mendes, Embrapa Recursos Genéticos.

RESUMO - A família Orchidaceae está distribuída em praticamente todas as regiões da Terra com uma representação grande no Brasil, onde ocorrem cerca de 190 gêneros e 2.300 espécies, sendo 233 espécies registradas no Distrito Federal. Pela beleza das flores, são fortes alvos de colecionadores e comerciantes que coletam espécimes até em áreas de reserva. Aliado à destruição dos seus hábitats pela expansão da ocupação do Cerrado, este extrativismo ameaça muitas espécies de extinção. A conservação de germoplasma em forma de sementes seria uma alternativa altamente interessante para estas espécies de orquídeas. No presente trabalho testou-se a conservação de sementes de algumas espécies de orquídeas do Cerrado: Cyrtopodium eugenii, C. paludicolum, C. inaldianum, C. saintlegerianum, Epidendrum nocturnum e Schomburgkia crispa. A viabilidade das sementes foi avaliada pelo teste de tetrazólio e, simultaneamente, conferida por germinação in vitro em meio $1 \frac{1}{2} \mathrm{MS}$. Para classificar o comportamento no armazenamento, as sementes foram dessecadas sobre sílica gel e mantidas por 72 horas nos seguintes tratamentos: $25^{\circ} \mathrm{C}, 5^{\circ} \mathrm{C},-20^{\circ} \mathrm{Ce}$ $-196^{\circ} \mathrm{C}$. Outras amostras foram armazenadas por períodos de 30, 180 e 360 dias sob as mesmas condições. Após estes períodos as sementes foram submetidas a testes de tetrazólio e de germinação para verificar a viabilidade. A porcentagem de sementes viáveis com base no teste de tetrazólio coincidiu com a porcentagem de germinação em todas as espécies nos tratamentos até 180 dias de armazenamento, com exceção de uma amostra. Após 360 dias de armazenamento, não houve correspondência entre os dois métodos de avaliação da viabilidade. Em todos os tratamentos, as sementes se conservaram sem perda de viabilidade por 72 horas, indicando um comportamento ortodoxo quanto ao armazenamento. Aos 180 ou 360 dias, dependendo da espécie e do acesso, todas as sementes armazenadas na temperatura ambiente haviam perdido sua viabilidade. De um modo geral, o armazenamento a $5^{\circ} \mathrm{C} \mathrm{e}-196^{\circ} \mathrm{C}$ manteve altas porcentagens de germinação (acima de 60\%) por um ano para Cyrtopodium eugenii, $C$. inaldianum, $C$. saintlegerianum, Epidendrum nocturnum e um acesso de Schomburgkia crispa. Nas outras espécies e amostras (Cyrtopodium paludicolum e dois acessos de Schomburgkia crispa) a porcentagem de germinação caiu para valores entre 20 e $40 \%$ após 360 dias de armazenamento. $\mathrm{O}$ armazenamento a $-20^{\circ} \mathrm{C}$ foi efetivo para algumas amostras mas causou maiores perdas de viabilidade do que os tratamentos de $5^{\circ} \mathrm{Ce}-196^{\circ} \mathrm{C}$ em três das espécies de Cyrtopodium. Os estudos demonstraram a possibilidade de conservação de sementes das espécies de orquídeas estudadas em bancos de germoplasma.

Palavras-chave-germinação; Orchidaceae; tetrazólio. 
TÍTULO:

AUTOR DA TESE:

DATA DA DEFESA:

LOCAL:
Arecaceae (Palmae) no Distrito Federal (Brasil)

Renata Corrêa Martins

$17 /$ fevereiro/ 2000

Universidade de Brasília, Departamento de Botânica, Brasília, DF.

Mestrado

BANCA EXAMINADORA: Dr. Tarciso Filgueiras (orientador), IBGE/UnB

Dr. Ângelo Rizzo, UFG

Dr. Aldicir Scariot, EMBRAPA-CENARGEN

RESUMO-Este trabalho consiste no levantamento das espécies nativas de Arecaceae (Palmae) ocorrentes no Distrito Federal (DF), Brasil. Foram encontrados 8 gêneros e 16 espécies. São elas: Acrocomia aculeata, Allagoptera campestris, Allagoptera leucocalyx, Attalea brasiliensis, Attalea geraensis, Attalea phalerata, Butia archeri, Euterpe edulis, Geonoma brevispatha, Geonoma schottiana vel aff., Mauritia flexuosa, Syagrus comosa, Syagrus flexuosa, Syagrus oleracea, Syagrus petraea, Syagrus romanzoffiana. Em vista de que muitas características diagnósticas importantes não eram encontradas nos materiais de herbário disponíveis para este estudo, foi elaborado um Roteiro Morfológico para ser utilizado durante as coletas de material botânico. São apresentadas descrições sucintas dos gêneros, descrições padronizadas das espécies, chaves para identificação dos gêneros e espécies, chave para identificação das espécies baseada em caracteres exclusivamente vegetativos, ilustrações de caracteres diagnósticos e mapas de distribuição no DF. As espécies encontram-se nas seguintes fitofisionomias: mata de galeria (10 spp.), campo sujo (7 ), campo limpo (6), cerrado s.s (6), cerradão (2), vereda (1). Dez espécies foram encontradas em ambientes antrópicos. São feitas duas citações de novas ocorrências para o Distrito Federal: Attalea geraensis, Butia archeri. Entretanto, ressalta-se que quase todo material de B.archeri havia sido citado na literatura como B.leiospatha. Registra-se pela primeira vez a ocorrência no DF de Attalea phalerata através de material botânico. As flores pistiladas de Attalea brasiliensis são aqui descritas pela primeira vez.

Palavras-chave-Arecaceae, Florística, Distrito Federal.

ABSTRACT - A floristic survey of the Arecaceae (Palmae) native to Brazil's Distrito Federal (DF) was undertaken. Eight genera and 16 species were found. They are: Acrocomia aculeata, Allagoptera campestris, Allagoptera leucocalyx, Attalea brasiliensis, Attalea geraensis, Attalea phalerata, Butia archeri, Euterpe edulis, Geonoma brevispatha, Geonoma schottiana vel aff., Mauritia flexuosa, Syagrus comosa, Syagrus flexuosa, Syagrus oleracea, Syagrus petraea, Syagrus romanzoffiana. Because some key diagnostic caracteristics were normally missing in the herbarium specimens available for this study, a field guide to collect palms specimens was designed. Keys and descriptions of genera and species, a key based solely on vegetative characteristics, illustrations of some key diagnostic features, and distribution maps are provided. The species are found in the following phytophisiognomies: gallery forest (10 spp.), campo sujo (7), campo limpo (6), cerrado s.s (6), cerradão (2), vereda (1). Ten species were found in anthropic habits. Two species are cited for the DF for the first time: Attalea geraensis, Butia archeri, but Butia archeri was previously cited as B. leiospatha. The occurence of Attalea phalerata is documented with herbarium material for the first time. The female flowers of Attalea brasiliensis are described for the first time.

Key word - Arecaceae, Floristic, Distrito Federal.

Agência(s) Financiadora(s): CAPES 